\title{
Prevalence of overweight and obesity in Mozambique in 2005 and 2015
}

\author{
Filipa Fontes ${ }^{1,2, *} \odot$, Albertino Damasceno ${ }^{1,3,4}$, Neusa Jessen ${ }^{1,3}$, António Prista ${ }^{5}$, \\ Carla Silva-Matos 6 , Patrícia Padrão ${ }^{1,7}$ and Nuno Lunet ${ }^{1,4}$
}

'EPIUnit - Instituto de Saúde Pública, Universidade do Porto, Rua das Taipas nº135, 4050-60 Porto, Portugal: ${ }^{2}$ Instituto Português de Oncologia do Porto, Porto, Portugal: ${ }^{3}$ Faculdade de Medicina, Universidade Eduardo Mondlane, Maputo, Mozambique: ${ }^{4}$ Departamento de Ciências da Saúde Pública e Forenses e Educação Médica, Faculdade de Medicina, Universidade do Porto, Porto, Portugal: ${ }^{5}$ Faculdade de Ciências de Educação Física e Desporto, Universidade Pedagógica de Maputo, Maputo, Mozambique: 'Unidade de Gestão do Fundo Global - Direção de Planificação e Cooperação, Ministério da Saúde de Moçambique, Maputo, Mozambique: ${ }^{7}$ Faculdade de Ciências da Nutrição e Alimentação, Universidade do Porto, Porto, Portugal

Submitted 21 September 2018: Final revision received 13 May 2019: Accepted 22 May 2019: First published online 27 August 2019

\begin{abstract}
Objective: The ongoing demographic, nutritional and epidemiological transitions in sub-Saharan Africa highlight the importance of monitoring overweight and obesity. We aimed to assess the prevalence of overweight and obesity in Mozambique in 2014/2015 and compare the estimates with those obtained in 2005. Design: Cross-sectional study conducted in 2014/2015, following the WHO Stepwise Approach to Chronic Disease Risk Factor Surveillance (STEPS). Prevalence estimates with $95 \%$ CI were computed for different categories of BMI and abdominal obesity, along with age-, education- and income-adjusted OR. The age-standardized prevalence in the age group 25-64 years was compared with results from a STEPS survey conducted in 2005.

Setting: Mozambique.

Participants: Representative sample of the population aged 18-64 years ( $n$ 2595). Results: Between 2005 and 2014/2015, the prevalence of overweight and obesity increased from 18.3 to $30.5 \%(P<0.001)$ in women and from 11.7 to $18.2 \%(P<0.001)$ in men. Abdominal obesity increased among women (from 9.4 to $20.4 \%, P<0.001)$, but there was no significant difference among men $(1.5 v$. $2 \cdot 1 \%, P=0 \cdot 395)$. In $2014 / 2015$, the prevalence of overweight and obesity was more than twofold higher in urban areas and in women; in the age group 18-24 years, it was highest in urban women and lowest in rural men.

Conclusions: In Mozambique, there was a steep increase in the prevalence of overweight and obesity among adults between 2005 and 2014/2015. Overweight and obesity are more prevalent in urban areas and among women, already affecting one in five urban women aged 18-24 years.
\end{abstract}

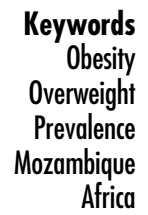

The widespread increase of the prevalence of overweight and obesity has long been described as a global pandemic $^{(1)}$. The total number of adults with overweight $\left(B M I \geq 25.0 \mathrm{~kg} / \mathrm{m}^{2}\right)$ and obesity $\left(\mathrm{BMI} \geq 30.0 \mathrm{~kg} / \mathrm{m}^{2}\right)$ was estimated to be 2.01 billion and 677 million, respectively, in $2016^{(2)}$. Overweight and obesity are well known risk factors for type 2 diabetes, hypertension, CVD and cancer ${ }^{(3)}$, accounting for an estimated $7 \cdot 1 \%$ of all deaths and $3.6 \%$ of years lived with disability among adults in $2015^{(4)}$.

The WHO Stepwise Approach to Chronic Disease Risk Factor Surveillance (STEPS) provides a standardized protocol for the surveillance of non-communicable diseases and their risk factors, including obesity, to guide monitoring efforts on a regular basis, mostly in low- and middle-income countries ${ }^{(5)}$. It is based on a sequential process, starting with the collection of key information on risk factors using a questionnaire, and moving on to simple physical measurements and collection of samples for biochemical analysis ${ }^{(5)}$. In Mozambique, the first WHO STEPS survey conducted in 2005 showed a low prevalence of obesity and overweight, although higher in urban areas and among women, mostly in those with higher 
socio-economic status ${ }^{(6)}$. According to these results, Mozambique was in stage 1 of the recently proposed conceptual model of the stages of the obesity transition ${ }^{(7)}$. However, an increase in the prevalence of obesity among adults, as well as a narrowing of the gap between genders and of the socio-economic differences among women may be expected, given the quick demographic, nutritional and epidemiological transitions occurring in this context. This highlights the importance of monitoring the prevalence of overweight and obesity, to guide local public health policies.

Therefore, the present study aimed to quantify the prevalence of overweight and obesity in the Mozambican adult population using data from the most recent STEPS survey conducted in Mozambique in 2014/2015, as well as to compare the estimates with those obtained in the 2005 STEPS evaluation.

\section{Methods}

The present study is based on a cross-sectional analysis of a representative sample of the Mozambican population aged 15-64 years, conducted between December 2014 and February 2015.

\section{Selection of participants}

The study design and implementation have been described in detail elsewhere ${ }^{(8)}$. Participants were selected through a complex sampling design, to obtain a representative sample at the national and provincial levels, and according to the place of residence (urban $v$. rural); the homeless and people living in collective residential institutions (e.g. hotels, hospitals, military facilities) were not eligible. Sampling procedures started with the selection of 120 primary sampling units (i.e. geographical units including 400 to 600 households), with a probability proportional to the number of households, stratified according to province, urban/rural areas and socio-economic strata. One enumeration area (i.e. geographical unit including eighty to 150 households) was then randomly selected within each primary sampling unit, and twenty-four households were randomly and systematically selected, from updated lists of households, in each enumeration area. A maximum of two participants were selected from each selected household, one aged 15-44 years and one aged 45-64 years, whenever available; whenever there was more than one household member in each of these age groups, only one per group was randomly selected using a Kish selection grid. A total of 3277 individuals were invited and 3119 (95.2\%) agreed to participate in the survey. Sampling weights were computed considering the number of participants evaluated in each stratum in relation to the number expected per stratum, according to the population projection for the same period. A total of 2756 participants were eligible for the present study, after excluding those below 18 years of age $(n 248)$ and pregnant women (n 115).

A STEPS survey was previously conducted in Mozambique in 2005, as described in detail elsewhere ${ }^{(6)}$. It was based on essentially the same methods used in 2014/2015 and the data set was available for comparison with the most recent data.

\section{Evaluation of the participants}

Participants were evaluated using standardized methods, following the WHO STEPS ${ }^{(5)}$. The Portuguese version of the WHO STEPS instrument for non-communicable disease risk factors (Core and Expanded version 3.0) was used by trained interviewers for data collection.

Anthropometric measurements were obtained with participants wearing light clothing and no footwear. Body weight was measured to the nearest $0 \cdot 1 \mathrm{~kg}$ using a digital scale and height to the nearest $0.1 \mathrm{~cm}$ in the standing position using a portable stadiometer. BMI was calculated as weight (in kilograms) divided by the square of height (in metres) and stratified into the categories: $<18.5 \mathrm{~kg} / \mathrm{m}^{2}$, $18.5-24.9 \mathrm{~kg} / \mathrm{m}^{2}, 25.0-29.9 \mathrm{~kg} / \mathrm{m}^{2}$ and $\geq 30.0 \mathrm{~kg} / \mathrm{m}^{2}$, corresponding to underweight, normal, overweight and obesity, respectively, as defined by the $\mathrm{WHO}^{(9)}$. Waist circumference was measured to the nearest $0 \cdot 1 \mathrm{~cm}$, using a constant tension tape, directly over the skin or over light clothing, at the level of the midpoint between the inferior margin of the last rib and the iliac crest in the mid-axillary line. Participants were classified as having abdominal obesity if their waist circumference was greater than 88 and $102 \mathrm{~cm}$ in women and men, respectively, as defined by the $\mathrm{WHO}^{(9)}$.

The classification of the place of residence as urban or rural and the definition of the highest level of education attained were conducted in accordance with the 2007 census $^{(10)}$. Monthly income per capita in meticais, per household member aged $\geq 18$ years, was converted into US dollars, taking into account the exchange rate in the midpoint of the time period of the participants' evaluation.

\section{Statistical analysis}

Means with $95 \%$ CI were computed for BMI and waist circumference, and prevalence estimates as percentages with $95 \%$ CI for different categories of BMI and waist circumference. Age-, education- and income-adjusted regression coefficients were computed using linear regression to estimate the strength of the association between sociodemographic characteristics and waist circumference, according to place of residence. Logistic regression was used to assess the association of the same characteristics with overweight and obesity. In addition, models including the same independent variables were fitted to quantify the association of place of residence and gender with overweight and obesity and abdominal obesity. 
Table 1 Sociodemographic characteristics of the participants: representative sample of the Mozambique population aged 18-64 years ( $n$ 2595), 2014/2015

\begin{tabular}{|c|c|c|c|c|c|c|}
\hline & \multicolumn{3}{|c|}{ Women (n 1554) } & \multicolumn{3}{|c|}{ Men $(n 1041)$} \\
\hline & $n$ & Unweighted (\%) & Weighted (\%) & $n$ & Unweighted (\%) & Weighted (\%) \\
\hline \multicolumn{7}{|c|}{ Place of residence } \\
\hline Urban & 756 & $48 \cdot 6$ & $41 \cdot 0$ & 464 & $44 \cdot 6$ & 33.9 \\
\hline Rural & 798 & $51 \cdot 4$ & $59 \cdot 0$ & 577 & $55 \cdot 4$ & $66 \cdot 1$ \\
\hline \multicolumn{7}{|c|}{ Age (years) } \\
\hline $18-24$ & 358 & 23.0 & $23 \cdot 4$ & 243 & $23 \cdot 3$ & $22 \cdot 7$ \\
\hline $25-34$ & 447 & $28 \cdot 8$ & 29.5 & 258 & $24 \cdot 8$ & $22 \cdot 1$ \\
\hline $35-44$ & 312 & $20 \cdot 1$ & $21 \cdot 1$ & 199 & $19 \cdot 1$ & $21 \cdot 6$ \\
\hline $45-54$ & 250 & $16 \cdot 1$ & $14 \cdot 1$ & 204 & $19 \cdot 6$ & $19 \cdot 6$ \\
\hline $55-64$ & 187 & $12 \cdot 0$ & 11.9 & 137 & $13 \cdot 2$ & $14 \cdot 0$ \\
\hline \multicolumn{7}{|c|}{ Education (years)* } \\
\hline 0 & 526 & 33.9 & $36 \cdot 3$ & 155 & 14.9 & $16 \cdot 7$ \\
\hline $1-5$ & 462 & $29 \cdot 8$ & $33 \cdot 0$ & 318 & $30 \cdot 6$ & 35.4 \\
\hline $6-7$ & 165 & $10 \cdot 6$ & $10 \cdot 0$ & 213 & $20 \cdot 5$ & $20 \cdot 8$ \\
\hline $8-10$ & 209 & 13.5 & 11.6 & 163 & $15 \cdot 7$ & $13 \cdot 7$ \\
\hline $11-12$ & 143 & $9 \cdot 2$ & $7 \cdot 3$ & 122 & 11.8 & 9.4 \\
\hline$\geq 13$ & 45 & 2.9 & $1 \cdot 8$ & 67 & $6 \cdot 4$ & 4.0 \\
\hline \multicolumn{7}{|c|}{ Monthly income ( $\$$ US/household member aged $\geq 18$ years) } \\
\hline $0-15$ & 371 & $28 \cdot 6$ & $33 \cdot 3$ & 254 & $26 \cdot 5$ & $33 \cdot 6$ \\
\hline $16-22$ & 230 & $17 \cdot 7$ & 19.5 & 143 & 14.9 & $18 \cdot 3$ \\
\hline $23-44$ & 287 & $22 \cdot 1$ & $20 \cdot 8$ & 154 & $16 \cdot 1$ & $14 \cdot 1$ \\
\hline $45-89$ & 202 & $15 \cdot 6$ & $13 \cdot 3$ & 170 & $17 \cdot 8$ & $15 \cdot 7$ \\
\hline$\geq 90$ & 209 & $16 \cdot 1$ & $13 \cdot 2$ & 236 & $24 \cdot 7$ & $18 \cdot 3$ \\
\hline
\end{tabular}

The 2005 results were compared with those observed in the current STEPS survey, after direct standardization using the 2005 population as reference. Since in 2005 only adults aged 25-64 years were evaluated, all comparisons with the 2014/2015 data referred to the same age strata. Hypothesis tests for no change between the two periods were conducted by dividing the difference in proportions or means estimated in the two surveys by its SE, and calculating a twosided $P$ value using the Student's $t$ distribution ${ }^{(11)}$. A $P$ value less than 0.05 was considered to be statistically significant.

Analyses were conducted using the statistical software package Stata version 11.2, considering the sampling weights, so that the computed estimates reflect the means and prevalence in Mozambique, and adjusting for stratification by province and clustering at the primary sampling unit level.

\section{Results}

A total of 2595 participants were considered for the present analysis, after excluding those from whom data on weight, height or waist circumference were unavailable ( $n$ 161). The latter were more often dwellers in urban areas than those included in the analysis $(57.1 \mathrm{v} .47 .0 \%$, $P=0.013$ ), but there were no statistically significant differences regarding gender (proportion of women: $55.8 \mathrm{v}$. $59.9 \%, P=0.317$ ), age (mean age: 34.6 v. 36.5 years, $P=0.077$ ) and education (proportion with $\geq 1$ year of education: $73.8 v .73 .7 \%, P=0.986)$.
In 2014/2015, just over a third of the population lived in urban areas and approximately half were aged 18-34 years. Approximately one in three women and one in six men had no formal education, and one-third reported a monthly income of less than \$US 16 per adult household member (Table 1).

\section{BMI in 2014/2015}

The mean BMI $\left(\mathrm{kg} / \mathrm{m}^{2}\right)$ was $23.5(95 \%$ CI $23.1,23.9)$ among women and 22.1 (95\% CI $21.7,22.5)$ in men. The prevalence of overweight was 17.5 (95\% CI $14.9,20 \cdot 1) \%$ and $12 \cdot 1$ (95\% CI 9.5, 14.7)\% among women and men, respectively, and higher in urban than rural areas in both genders (Table 2). Concerning obesity, the prevalence was $10 \cdot 4$ $(95 \% \mathrm{CI} 8.4,12.5) \%$ among women and $4.2(95 \% \mathrm{CI}$ $2 \cdot 6,5 \cdot 8) \%$ in men, also higher in urban settings and among women. Underweight affected approximately one in ten participants, in both genders and settings (Table 2).

In rural areas, in both women and men, there was a tendency for overweight and obesity prevalence to increase with age. Among those aged 18-24 years, the prevalence of overweight and obesity was highest in urban women $(22.0 \%)$ and lowest in rural men (5.8\%; Table 3). The prevalence of overweight and obesity was highest among women (urban, 44.4\%; rural, 21.4\%) and urban men $(32.8 \%)$ with $1-5$ years of education, and in the more educated rural men (12.4\%). In men, in both settings, the prevalence of overweight and obesity increased with income, while no such tendency was observed among women (Table 3). 
The association between place of residence and overweight and obesity was attenuated after adjustments for age, education and income. The association between gender and overweight and obesity was stronger after adjustment for sociodemographic characteristics (Table 4).

\section{Waist circumference in 2014/2015}

The mean waist circumference (cm) was 78.1 (95\% CI 77.4, 78.9) among women and 76.6 (95\% CI 75.8, 77.4) in men, and higher in urban areas. The prevalence of abdominal obesity was 16.5 (95\% CI 13.8, 19.3) \% among women and $1.8(95 \%$ CI $0.9,2.7) \%$ in men, and was also higher in urban settings (Table 2).

There was an increase of waist circumference with age, except among the oldest women in urban areas. The differences between settings were highest at age 45-54 years in both genders; in relation to rural dwellers, the mean waist circumference of urban women and men was 9.9 and $7.4 \mathrm{~cm}$ larger, respectively (Table 3 ).

Waist circumference was highest in urban and rural women, and in urban men with $1-5$ years of education. In men, it increased with income (Table 3).

The prevalence of abdominal obesity was higher in urban than rural areas. The relationship between gender and abdominal obesity was stronger after adjustments for age, education and income, in a similar extent in both settings (Table 4).

\section{Comparison between the results obtained in the 2005 and 2014/2015 surveys}

Between 2005 and 2015, there were increases in mean BMI and mean waist circumference. The prevalence of overweight and obesity increased from 18.3 to $30.5 \%$ among women and from 11.7 to $18.2 \%$ in men. For obesity, there was an increase from 7.0 to $13.0 \%$ among women and from 2.3 to $5.0 \%$ in men. The prevalence of abdominal obesity in $2014 / 2015$ was more than twofold higher among women (Table 5)

\section{Discussion}

In Mozambique, the prevalence of obesity and overweight increased significantly in just a decade. In 2014/2015, almost one in five men and one in three women were affected. Among the latter, there was a twofold higher prevalence of abdominal obesity than 10 years before, being observed in one out of five women in the most recent years. The prevalence of overweight/obesity was more than twice as high in urban than rural areas.

Previous studies analysing overweight and obesity trends worldwide showed a slowing down in the rate of increase of the prevalence of overweight and obesity in high-income countries and a more rapid increase in the proportion of those living with these conditions in low- 


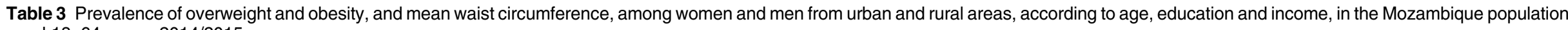
aged 18-64 years, 2014/2015

\begin{tabular}{|c|c|c|c|c|c|c|c|c|c|c|c|c|c|c|c|c|}
\hline & \multicolumn{16}{|c|}{ Overweight and obesity $\left(\mathrm{BMI} \geq 25.0 \mathrm{~kg} / \mathrm{m}^{2}\right)$} \\
\hline & \multicolumn{8}{|c|}{ Women } & \multicolumn{8}{|c|}{ Men } \\
\hline & \multicolumn{4}{|c|}{ Urban } & \multicolumn{4}{|c|}{ Rural } & \multicolumn{4}{|c|}{ Urban } & \multicolumn{4}{|c|}{ Rural } \\
\hline & $\%$ & $95 \% \mathrm{Cl}$ & $\mathrm{OR}^{*}$ & $95 \% \mathrm{Cl}$ & $\%$ & $95 \% \mathrm{Cl}$ & $\mathrm{OR}^{*}$ & $95 \% \mathrm{Cl}$ & $\%$ & $95 \% \mathrm{Cl}$ & $\mathrm{OR}^{*}$ & $95 \% \mathrm{Cl}$ & $\%$ & $95 \% \mathrm{Cl}$ & $\mathrm{OR}^{*}$ & $95 \% \mathrm{Cl}$ \\
\hline \multicolumn{17}{|c|}{ Age (years) } \\
\hline $18-24$ & $22 \cdot 0$ & $14 \cdot 2,29 \cdot 8$ & 0.51 & $0.27,0.98$ & $15 \cdot 1$ & $7 \cdot 9,22 \cdot 2$ & 0.74 & $0.39,1.42$ & $11 \cdot 9$ & $3 \cdot 0,20 \cdot 7$ & 0.46 & $0.20,1.07$ & $5 \cdot 8$ & $1 \cdot 3,10 \cdot 3$ & 0.65 & $0.25,1.73$ \\
\hline $25-34$ & $38 \cdot 3$ & $30 \cdot 5,46 \cdot 0$ & 1.00 & Ref. & 17.5 & $11 \cdot 4,23 \cdot 4$ & 1.00 & Ref. & $27 \cdot 3$ & $18 \cdot 8,35 \cdot 8$ & 1.00 & Ref. & 11.5 & $6 \cdot 2,16 \cdot 8$ & 1.00 & Ref. \\
\hline $35-44$ & $55 \cdot 2$ & $44.8,65 \cdot 5$ & 1.93 & $1.22,3.05$ & $17 \cdot 1$ & $11 \cdot 7,22 \cdot 6$ & 1.06 & $0.57,1.95$ & $25 \cdot 6$ & $11.6,39.6$ & 0.98 & $0.40,2.38$ & 11.9 & $3 \cdot 4,20 \cdot 3$ & 1.33 & $0.51,3.43$ \\
\hline $45-54$ & $57 \cdot 6$ & $46 \cdot 4,68 \cdot 8$ & $2 \cdot 83$ & $1.47,5.45$ & $24 \cdot 1$ & $14 \cdot 7,33 \cdot 4$ & $1 \cdot 18$ & $0.62,2.25$ & $40 \cdot 2$ & $28 \cdot 6,51 \cdot 8$ & 1.78 & $0.89,3.55$ & $12 \cdot 2$ & $4 \cdot 4,20 \cdot 0$ & 1.46 & $0.70,3.04$ \\
\hline $55-64$ & 44.4 & $28 \cdot 0,60 \cdot 8$ & 1.55 & $0.50,4.81$ & $30 \cdot 2$ & $20 \cdot 9,39.5$ & 2.49 & $1.29,4.83$ & 39.4 & $25 \cdot 5,53 \cdot 4$ & 1.97 & $0.93,4.18$ & 14.9 & $7 \cdot 3,22 \cdot 6$ & 2.06 & $0 \cdot 75,5 \cdot 71$ \\
\hline \multicolumn{17}{|c|}{ Education (years) } \\
\hline$<1$ & $35 \cdot 3$ & $27 \cdot 8,42 \cdot 8$ & 1.00 & Ref. & $17 \cdot 1$ & $11 \cdot 6,22 \cdot 6$ & 1.00 & Ref. & 20.5 & $6 \cdot 7,34 \cdot 3$ & 1.00 & Ref. & $12 \cdot 2$ & $5 \cdot 6,18 \cdot 8$ & 1.00 & Ref. \\
\hline $1-5$ & 44.4 & $35 \cdot 0,53 \cdot 7$ & $2 \cdot 00$ & $1 \cdot 22,3 \cdot 27$ & 21.4 & $14 \cdot 6,28 \cdot 2$ & 1.40 & $0.74,2 \cdot 67$ & $32 \cdot 8$ & $14 \cdot 4,51 \cdot 1$ & 1.84 & $0.51,6.61$ & $9 \cdot 3$ & $4 \cdot 2,14 \cdot 4$ & 0.72 & $0.30,1.74$ \\
\hline$\geq 6$ & 40.4 & $33.9,47 \cdot 0$ & 1.84 & $0.89,3.81$ & $21 \cdot 0$ & $15 \cdot 3,26 \cdot 8$ & 1.60 & $0.88,2.92$ & $25 \cdot 1$ & $18 \cdot 6,31 \cdot 6$ & 1.44 & $0.47,4.40$ & $12 \cdot 4$ & $6 \cdot 5,18 \cdot 3$ & 1.00 & $0.35,2.83$ \\
\hline \multicolumn{17}{|c|}{ Monthly income ( $\$$ US/household member aged $\geq 18$ years) } \\
\hline $0-15$ & 39.6 & $30 \cdot 8,48 \cdot 3$ & 1.00 & Ref. & $15 \cdot 4$ & $10 \cdot 5,20 \cdot 3$ & 1.00 & Ref. & $12 \cdot 9$ & $3 \cdot 6,22 \cdot 1$ & 1.00 & Ref. & 6.9 & $3 \cdot 8,10 \cdot 1$ & 1.00 & Ref. \\
\hline $16-44$ & $36 \cdot 1$ & $26 \cdot 9,45 \cdot 2$ & 0.78 & $0.44,1.37$ & $24 \cdot 1$ & $17 \cdot 8,30.5$ & 1.75 & $1 \cdot 09,2 \cdot 81$ & $22 \cdot 8$ & $13 \cdot 0,32 \cdot 6$ & 1.92 & $0.75,4.92$ & $12 \cdot 6$ & $7 \cdot 8,17 \cdot 4$ & 2.05 & $1.17,3.58$ \\
\hline$\geq 45$ & $51 \cdot 0$ & $42 \cdot 3,59 \cdot 7$ & 1.52 & $0.92,2.52$ & $22 \cdot 2$ & $16 \cdot 3,28 \cdot 0$ & 1.47 & $0.86,2.51$ & $35 \cdot 6$ & $29 \cdot 5,41 \cdot 8$ & 3.20 & $1.31,7.81$ & $18 \cdot 4$ & $10 \cdot 7,26 \cdot 1$ & $3 \cdot 24$ & $1 \cdot 69,6 \cdot 21$ \\
\hline
\end{tabular}

Waist circumference $(\mathrm{cm})$

\begin{tabular}{|c|c|c|c|c|c|c|c|c|c|c|c|c|c|c|c|c|}
\hline & \multicolumn{16}{|c|}{ Waist circumference $(\mathrm{cm})$} \\
\hline & \multicolumn{8}{|c|}{ Women } & \multicolumn{8}{|c|}{ Men } \\
\hline & \multicolumn{4}{|c|}{ Urban } & \multicolumn{4}{|c|}{ Rural } & \multicolumn{4}{|c|}{ Urban } & \multicolumn{4}{|c|}{ Rural } \\
\hline & Mean & $95 \% \mathrm{Cl}$ & $\begin{array}{c}\text { Mean } \\
\text { difference }{ }^{\star}, \dagger\end{array}$ & $95 \% \mathrm{Cl}$ & Mean & $95 \% \mathrm{Cl}$ & $\begin{array}{c}\text { Mean } \\
\text { difference }^{*}, \dagger\end{array}$ & $95 \% \mathrm{Cl}$ & Mean & $95 \% \mathrm{Cl}$ & $\begin{array}{c}\text { Mean } \\
\text { difference }{ }^{\star}, \dagger\end{array}$ & $95 \% \mathrm{Cl}$ & Mean & $95 \% \mathrm{Cl}$ & $\begin{array}{c}\text { Mean } \\
\text { difference }{ }^{\star}, \dagger\end{array}$ & $95 \% \mathrm{Cl}$ \\
\hline \multicolumn{17}{|c|}{ Age (years) } \\
\hline $18-24$ & 73.3 & $71 \cdot 7,74 \cdot 8$ & -7.46 & $-10 \cdot 1,-4.78$ & $74 \cdot 1$ & $72 \cdot 7,75 \cdot 4$ & -2.00 & $-4 \cdot 17,0 \cdot 18$ & $72 \cdot 4$ & $71 \cdot 1,73 \cdot 8$ & -3.03 & $-5.93,-0.12$ & $73 \cdot 1$ & $72 \cdot 0,74 \cdot 1$ & -1.11 & $-2.53,0.31$ \\
\hline $25-34$ & 80.5 & $78 \cdot 0,83 \cdot 1$ & 0.00 & Ref. & $76 \cdot 0$ & $74 \cdot 7,77 \cdot 3$ & 0.00 & Ref. & 76.5 & $74 \cdot 3,78 \cdot 7$ & 0.00 & Ref. & 74.6 & $73 \cdot 4,75 \cdot 7$ & 0.00 & Ref. \\
\hline $35-44$ & $82 \cdot 8$ & $79.9,85 \cdot 8$ & $2 \cdot 30$ & $-1 \cdot 00,5 \cdot 60$ & $77 \cdot 0$ & $75 \cdot 0,78.5$ & 1.69 & $-0.38,3.75$ & $79 \cdot 1$ & $76 \cdot 7,81.5$ & 4.24 & $1 \cdot 16,7.32$ & $75 \cdot 7$ & $73 \cdot 2,78 \cdot 1$ & 1.90 & $-1 \cdot 10,4 \cdot 91$ \\
\hline $45-54$ & 87.5 & $84 \cdot 3,90 \cdot 8$ & 8.48 & $4.93,12 \cdot 0$ & $77 \cdot 6$ & $75 \cdot 1,80 \cdot 1$ & 1.27 & $-1 \cdot 87,4 \cdot 40$ & $84 \cdot 1$ & $81 \cdot 6,85 \cdot 6$ & $8 \cdot 17$ & $5.02,11 \cdot 3$ & $76 \cdot 7$ & $74 \cdot 7,78 \cdot 6$ & $3 \cdot 29$ & $1 \cdot 27,5 \cdot 32$ \\
\hline $55-64$ & 83.1 & $80 \cdot 3,85 \cdot 9$ & 4.55 & $0.43,8.68$ & $80 \cdot 6$ & $78.4,82.9$ & $5 \cdot 67$ & $3.00,8.34$ & $86 \cdot 5$ & $82 \cdot 8,90 \cdot 2$ & 11.40 & $7 \cdot 88,14.92$ & $79 \cdot 2$ & $77 \cdot 2,81 \cdot 3$ & $6 \cdot 30$ & $3.07,9.54$ \\
\hline \multicolumn{17}{|c|}{ Education (years) } \\
\hline$<1$ & $79 \cdot 6$ & $77 \cdot 9,81 \cdot 4$ & 0.00 & Ref. & $76 \cdot 9$ & $75 \cdot 8,78 \cdot 0$ & 0.00 & Ref. & $80 \cdot 0$ & $74 \cdot 1,86 \cdot 0$ & 0.00 & Ref. & $75 \cdot 7$ & $74 \cdot 0,77 \cdot 3$ & 0.00 & Ref. \\
\hline $1-5$ & 81.4 & $78 \cdot 7,84.1$ & $2 \cdot 84$ & $-0.13,5.81$ & $77 \cdot 1$ & $75 \cdot 9,78.4$ & 1.15 & $-0.74,3.04$ & 78.4 & $76 \cdot 5,80 \cdot 4$ & -2.03 & $-8.43,4.37$ & $75 \cdot 1$ & $73 \cdot 6,76 \cdot 6$ & -0.78 & $-2.57,1.00$ \\
\hline$\geq 6$ & $80 \cdot 1$ & $78 \cdot 2,82 \cdot 0$ & 3.88 & $1.02,6.74$ & 74.7 & $72 \cdot 9,78 \cdot 4$ & -0.08 & $-2 \cdot 56,2 \cdot 40$ & $78 \cdot 3$ & $77 \cdot 2,79 \cdot 4$ & 0.18 & $-6 \cdot 37,6 \cdot 73$ & $76 \cdot 2$ & $74 \cdot 9,77 \cdot 6$ & 1.50 & $-0.91,3.91$ \\
\hline \multicolumn{17}{|c|}{ Monthly income (\$US/household member aged $\geq 18$ years) } \\
\hline $0-15$ & 80.8 & $78 \cdot 4,83.2$ & 0.00 & Ref. & $75 \cdot 8$ & $74.6,77.0$ & 0.00 & Ref. & $74 \cdot 2$ & $71.9,76 \cdot 6$ & 0.00 & Ref. & 74.5 & $72 \cdot 9,76 \cdot 2$ & 0.00 & Ref. \\
\hline $16-44$ & 79.2 & $76 \cdot 7,81 \cdot 8$ & $-2 \cdot 01$ & $-5 \cdot 12,1 \cdot 11$ & 77.4 & $75 \cdot 8,79 \cdot 0$ & 1.72 & $-0.18,3.63$ & 77.0 & $75.4,78.5$ & 2.05 & $-0.14,4.24$ & 75.4 & $74.4,76 \cdot 5$ & $1 \cdot 13$ & $-1 \cdot 00,3 \cdot 26$ \\
\hline$\geq 45$ & $82 \cdot 3$ & $80 \cdot 1,84.5$ & 1.02 & $-1 \cdot 75,3 \cdot 79$ & $77 \cdot 3$ & $75 \cdot 5,79 \cdot 1$ & 1.73 & $-0.43,3.88$ & 81.6 & $79 \cdot 5,83 \cdot 6$ & $5 \cdot 66$ & $3.39,7.93$ & 78.0 & $76 \cdot 2,79 \cdot 8$ & 3.63 & $1 \cdot 12,6 \cdot 13$ \\
\hline
\end{tabular}

\begin{tabular}{|c|c|c|c|c|c|c|c|c|c|c|c|c|c|c|c|c|}
\hline & \multicolumn{16}{|c|}{ Waist circumference $(\mathrm{cm})$} \\
\hline & \multicolumn{8}{|c|}{ Women } & \multicolumn{8}{|c|}{ Men } \\
\hline & \multicolumn{4}{|c|}{ Urban } & \multicolumn{4}{|c|}{ Rural } & \multicolumn{4}{|c|}{ Urban } & \multicolumn{4}{|c|}{ Rural } \\
\hline & Mean & $95 \% \mathrm{Cl}$ & $\begin{array}{c}\text { Mean } \\
\text { difference }{ }^{*}, \dagger\end{array}$ & $95 \% \mathrm{Cl}$ & Mean & $95 \% \mathrm{Cl}$ & $\begin{array}{c}\text { Mean } \\
\text { difference }{ }^{\star}, \dagger\end{array}$ & $95 \% \mathrm{Cl}$ & Mean & $95 \% \mathrm{Cl}$ & $\begin{array}{c}\text { Mean } \\
\text { difference }{ }^{*}, \dagger\end{array}$ & $95 \% \mathrm{Cl}$ & Mean & $95 \% \mathrm{Cl}$ & $\begin{array}{c}\text { Mean } \\
\text { difference* }, \dagger\end{array}$ & $95 \% \mathrm{Cl}$ \\
\hline \multicolumn{17}{|c|}{ Age (years) } \\
\hline $18-24$ & $73 \cdot 3$ & $71 \cdot 7,74 \cdot 8$ & -7.46 & $-10 \cdot 1,-4 \cdot 78$ & 74.1 & $72 \cdot 7,75 \cdot 4$ & -2.00 & $-4 \cdot 17,0 \cdot 18$ & $72 \cdot 4$ & $71 \cdot 1,73 \cdot 8$ & -3.03 & $-5.93,-0.12$ & $73 \cdot 1$ & $72 \cdot 0,74 \cdot 1$ & -1.11 & $-2.53,0.31$ \\
\hline $25-34$ & 80.5 & $78 \cdot 0,83.1$ & 0.00 & Ref. & $76 \cdot 0$ & $74 \cdot 7,77 \cdot 3$ & 0.00 & Ref. & 76.5 & $74.3,78 \cdot 7$ & 0.00 & Ref. & 74.6 & $73 \cdot 4,75 \cdot 7$ & 0.00 & Ref. \\
\hline $35-44$ & $82 \cdot 8$ & $79.9,85 \cdot 8$ & $2 \cdot 30$ & $-1.00,5.60$ & $77 \cdot 0$ & $75 \cdot 0,78.5$ & 1.69 & $-0.38,3.75$ & $79 \cdot 1$ & $76 \cdot 7,81 \cdot 5$ & $4 \cdot 24$ & $1 \cdot 16,7.32$ & $75 \cdot 7$ & $73 \cdot 2,78 \cdot 1$ & 1.90 & $-1.10,4.91$ \\
\hline $45-54$ & 87.5 & $84.3,90 \cdot 8$ & 8.48 & $4.93,12 \cdot 0$ & $77 \cdot 6$ & $75 \cdot 1,80 \cdot 1$ & 1.27 & $-1 \cdot 87,4.40$ & $84 \cdot 1$ & $81 \cdot 6,85 \cdot 6$ & $8 \cdot 17$ & $5 \cdot 02,11 \cdot 3$ & $76 \cdot 7$ & $74.7,78 \cdot 6$ & $3 \cdot 29$ & $1 \cdot 27,5 \cdot 32$ \\
\hline $55-64$ & $83 \cdot 1$ & $80 \cdot 3,85 \cdot 9$ & 4.55 & $0.43,8.68$ & $80 \cdot 6$ & $78 \cdot 4,82 \cdot 9$ & $5 \cdot 67$ & $3.00,8.34$ & $86 \cdot 5$ & $82 \cdot 8,90 \cdot 2$ & 11.40 & $7 \cdot 88,14.92$ & $79 \cdot 2$ & $77 \cdot 2,81 \cdot 3$ & $6 \cdot 30$ & $3.07,9.54$ \\
\hline \multicolumn{17}{|c|}{ Education (years) } \\
\hline$<1$ & $79 \cdot 6$ & $77 \cdot 9,81 \cdot 4$ & 0.00 & Ref. & $76 \cdot 9$ & $75 \cdot 8,78 \cdot 0$ & 0.00 & Ref. & $80 \cdot 0$ & $74 \cdot 1,86 \cdot 0$ & 0.00 & Ref. & 75.7 & $74 \cdot 0,77 \cdot 3$ & 0.00 & Ref. \\
\hline $1-5$ & 81.4 & $78 \cdot 7,84 \cdot 1$ & $2 \cdot 84$ & $-0.13,5.81$ & $77 \cdot 1$ & $75 \cdot 9,78 \cdot 4$ & 1.15 & $-0.74,3.04$ & 78.4 & $76 \cdot 5,80 \cdot 4$ & -2.03 & $-8.43,4.37$ & $75 \cdot 1$ & $73 \cdot 6,76 \cdot 6$ & -0.78 & $-2.57,1.00$ \\
\hline$\geq 6$ & $80 \cdot 1$ & $78 \cdot 2,82 \cdot 0$ & 3.88 & $1.02,6.74$ & 74.7 & $72 \cdot 9,78 \cdot 4$ & -0.08 & $-2 \cdot 56,2 \cdot 40$ & $78 \cdot 3$ & $77 \cdot 2,79 \cdot 4$ & 0.18 & $-6 \cdot 37,6.73$ & $76 \cdot 2$ & $74 \cdot 9,77 \cdot 6$ & 1.50 & $-0.91,3.91$ \\
\hline \multicolumn{17}{|c|}{ Monthly income ( $\$$ US/household member aged $\geq 18$ years) } \\
\hline $0-15$ & 80.8 & $78 \cdot 4,83 \cdot 2$ & 0.00 & Ref. & $75 \cdot 8$ & $74.6,77.0$ & 0.00 & Ref. & 74.2 & $71.9,76 \cdot 6$ & 0.00 & Ref. & 74.5 & $72 \cdot 9,76 \cdot 2$ & 0.00 & Ref. \\
\hline $16-44$ & $79 \cdot 2$ & $76 \cdot 7,81 \cdot 8$ & $-2 \cdot 01$ & $-5 \cdot 12,1 \cdot 11$ & $77 \cdot 4$ & $75 \cdot 8,79 \cdot 0$ & 1.72 & $-0.18,3.63$ & 77.0 & $75 \cdot 4,78.5$ & 2.05 & $-0.14,4.24$ & 75.4 & $74.4,76 \cdot 5$ & 1.13 & $-1.00,3.26$ \\
\hline$\geq 45$ & $82 \cdot 3$ & $80 \cdot 1,84.5$ & 1.02 & $-1.75,3.79$ & $77 \cdot 3$ & $75 \cdot 5,79 \cdot 1$ & 1.73 & $-0.43,3.88$ & 81.6 & $79.5,83 \cdot 6$ & $5 \cdot 66$ & $3.39,7.93$ & 78.0 & $76 \cdot 2,79 \cdot 8$ & 3.63 & $1 \cdot 12,6 \cdot 13$ \\
\hline
\end{tabular}

Ref., reference category.

(1)

Difference between the mean value of each category and the mean value of the reference category. 
Table $4 \mathrm{OR}$ and $95 \% \mathrm{Cl}$ for the association between place of residence and overweight and obesity, and abdominal obesity, according to place of residence and gender, in the Mozambique population aged 18-64 years, 2014/2015

\begin{tabular}{|c|c|c|c|c|c|c|c|c|}
\hline & \multicolumn{4}{|c|}{ Overweight and obesity (BMI $\left.\geq 25.0 \mathrm{~kg} / \mathrm{m}^{2}\right)$} & \multicolumn{4}{|c|}{ Abdominal obesity* } \\
\hline & Crude OR & $95 \% \mathrm{Cl}$ & Adjusted OR† & $95 \% \mathrm{Cl}$ & Crude OR & $95 \% \mathrm{Cl}$ & Adjusted OR $\ddagger$ & $95 \% \mathrm{Cl}$ \\
\hline \multicolumn{9}{|l|}{ Women } \\
\hline Rural & 1.00 & Ref. & 1.00 & Ref. & 1.00 & Ref. & 1.00 & Ref. \\
\hline Urban & $2 \cdot 86$ & $2 \cdot 00,4 \cdot 10$ & 2.49 & $1.74,3.58$ & 3.11 & $2 \cdot 09,4.61$ & $2 \cdot 62$ & $1.73,3.98$ \\
\hline \multicolumn{9}{|l|}{ Men } \\
\hline Rural & 1.00 & Ref. & 1.00 & Ref. & 1.00 & Ref. & 1.00 & Ref. \\
\hline Urban & $2 \cdot 87$ & $1 \cdot 79,4 \cdot 60$ & $2 \cdot 51$ & $1.56,4.02$ & 3.63 & $1 \cdot 22,10 \cdot 85$ & 3.36 & $1.06,10.65$ \\
\hline \multicolumn{9}{|l|}{ Urban } \\
\hline Men & 1.00 & Ref. & 1.00 & Ref. & 1.00 & Ref. & 1.00 & Ref. \\
\hline Women & 1.89 & $1.37,2.60$ & 2.34 & $1.62,3.37$ & 9.86 & $5.54,17.54$ & $15 \cdot 35$ & $8.13,28.99$ \\
\hline \multicolumn{9}{|l|}{ Rural } \\
\hline Men & 1.00 & Ref. & 1.00 & Ref. & 1.00 & Ref. & 1.00 & Ref. \\
\hline Women & 1.90 & $1.36,2.64$ & 2.35 & $1.66,3.33$ & 11.53 & $4.59,29.00$ & $19 \cdot 68$ & $6 \cdot 92,56 \cdot 01$ \\
\hline
\end{tabular}

Ref., reference category.

*Defined as waist circumference greater than 88 and $102 \mathrm{~cm}$ in women and men, respectively.

†Derived from models including age, education and income; $P_{\text {interaction }}=0.980$.

‡Derived from models including age, education and income; $P_{\text {interaction }}=0.681$.

Table 5 Overweight and abdominal obesity prevalence in Mozambique in 2005 and 2014/2015, among the population aged 25-64 years*

\begin{tabular}{|c|c|c|c|c|c|c|c|c|c|c|}
\hline & \multicolumn{5}{|c|}{ Women } & \multicolumn{5}{|c|}{ Men } \\
\hline & \multicolumn{4}{|c|}{ Survey year } & \multirow[b]{3}{*}{$\begin{array}{c}P \\
\text { value }\end{array}$} & \multicolumn{4}{|c|}{ Survey year } & \multirow[b]{3}{*}{$\begin{array}{c}P \\
\text { value }\end{array}$} \\
\hline & \multicolumn{2}{|c|}{2005} & \multicolumn{2}{|c|}{$2014 / 2015$} & & \multicolumn{2}{|c|}{2005} & \multicolumn{2}{|c|}{$2014 / 2015$} & \\
\hline & $\begin{array}{l}\text { Mean } \\
\text { or } \%\end{array}$ & $95 \% \mathrm{Cl}$ & $\begin{array}{l}\text { Mean } \\
\text { or } \%\end{array}$ & $95 \% \mathrm{Cl}$ & & $\begin{array}{l}\text { Mean } \\
\text { or } \%\end{array}$ & $95 \% \mathrm{Cl}$ & $\begin{array}{l}\text { Mean } \\
\text { or } \%\end{array}$ & $95 \% \mathrm{Cl}$ & \\
\hline BMI $\left(\mathrm{kg} / \mathrm{m}^{2}\right)$, mean and $95 \% \mathrm{Cl}$ & $22 \cdot 4$ & $21 \cdot 8,22 \cdot 9$ & 23.9 & $23 \cdot 5,24 \cdot 3$ & $<0.001$ & 21.4 & $21 \cdot 0,21 \cdot 8$ & $22 \cdot 2$ & $21 \cdot 9,22 \cdot 5$ & 0.002 \\
\hline $\begin{array}{l}\text { Waist circumference }(\mathrm{cm}) \text {, mean } \\
\text { and } 95 \% \mathrm{Cl}\end{array}$ & $75 \cdot 1$ & $74 \cdot 2,75 \cdot 9$ & 79.4 & $78 \cdot 6,80 \cdot 2$ & $<0.001$ & $76 \cdot 1$ & $75 \cdot 0,77 \cdot 3$ & 77.4 & $76 \cdot 6,78 \cdot 2$ & 0.069 \\
\hline \multicolumn{11}{|l|}{ Prevalence, $\%$ and $95 \% \mathrm{Cl}$} \\
\hline Obesity $\left(\mathrm{BMI} \geq 30.0 \mathrm{~kg} / \mathrm{m}^{2}\right)$ & $7 \cdot 0$ & $5 \cdot 2,8 \cdot 8$ & $13 \cdot 0$ & $10 \cdot 8,15 \cdot 2$ & $<0.001$ & $2 \cdot 3$ & $1 \cdot 1,3 \cdot 6$ & $5 \cdot 0$ & $3 \cdot 3,6 \cdot 6$ & 0.181 \\
\hline $\begin{array}{l}\text { Overweight and obesity } \\
\left(\mathrm{BMI} \geq 25.0 \mathrm{~kg} / \mathrm{m}^{2}\right)\end{array}$ & $18 \cdot 3$ & $13 \cdot 6,23 \cdot 0$ & 30.5 & $27 \cdot 2,33 \cdot 8$ & $<0.001$ & $11 \cdot 7$ & $7 \cdot 8,15 \cdot 7$ & $18 \cdot 2$ & $15 \cdot 1,21 \cdot 4$ & 0.012 \\
\hline Abdominal obesity $\dagger$ & $9 \cdot 4$ & $7 \cdot 0,11 \cdot 9$ & $20 \cdot 4$ & $17 \cdot 7,23 \cdot 1$ & $<0.001$ & 1.5 & $0.6,2.4$ & $2 \cdot 1$ & $1 \cdot 1,3 \cdot 2$ & 0.395 \\
\hline
\end{tabular}

${ }^{*}$ Results are presented as mean or as percentage, with $95 \% \mathrm{Cl}$, as specified.

†Defined as waist circumference greater than 88 and $102 \mathrm{~cm}$ in women and men, respectively.

and middle-income countries ${ }^{(12,13)}$. In sub-Saharan Africa, nationally representative data of overweight or obesity trends are scarce and focus mainly on women of childbearing age ${ }^{(14-16)}$. Nevertheless, findings from these studies showed that, in the past two decades, the prevalence of overweight and obesity more than doubled in most sub-Saharan African countries with data available ${ }^{(14,15)}$, being higher among women and urban residents ${ }^{(15,16)}$, which is in accordance with our results.

The trend towards the urbanization of the Mozambican population is likely to have contributed to the increase in the prevalence of overweight and obesity observed in the present study. The estimated urban Mozambican population was about $30 \%$ in 2005 and $34 \%$ in $2015^{(17)}$, being projected to increase to $37 \%$ by $2030^{(18)}$. Furthermore, a transition from a predominantly rural environment to a more urban 'westernized' society may have occurred within regions formally classified either as urban or as rural. Urbanization is associated with lifestyle changes, such as an increased consumption of energy-dense ultra-processed foods and sugar-sweetened beverages, along with a decrease in physical activity levels ${ }^{(1)}$. In 2005 , most adult Mozambicans were classified as having a high physical activity level ${ }^{(19)}$, according to the WHO criteria ${ }^{(20)}$; however, the prevalence of low level of physical activity was higher in urban areas and increased with education ${ }^{(19)}$. A shift towards more sedentary behaviours may have occurred over the past years, as a result of increasing urbanization and economic development, at least in those previously living in rural settings. Among Mozambicans, the use of motor vehicles and computer and television ownership increased between 2008/2009 and 2014/2015, from 5.6 to $12.2 \%$, from 1.5 to $5.6 \%$ and from 12.4 to $24 \cdot 2 \%$, respectively ${ }^{(21)}$. Also, a decline in physical activity 
was previously reported for children and adolescents ${ }^{(22)}$. In addition to the decrease in energy expenditure, the acquisition of new dietary habits, as demonstrated by the steep increase in the proportion of meals consumed outside the home $^{(23)}$ and the greater availability of fast-food restaurants and street food including fried and sweet meals ${ }^{(24)}$, may have contributed to the pattern found. Furthermore, the consumption of vegetables and fruit, known to contribute to the prevention of obesity and other non-communicable diseases, tends to decrease with urbanization, as reflected in the lower consumption of those foods in urban compared with rural areas in Mozambique ${ }^{(25)}$.

Young adults are in a period of transition from adolescence to adulthood, and therefore increasing decisionmaking autonomy, which makes them a vulnerable group for the acquisition of unhealthy lifestyles that may lead to overweight and obesity ${ }^{(26)}$. Despite the 2005 STEPS survey lacking data for the 18-24 years age group, precluding comparisons among younger individuals, in 2014/2015 the prevalence in this age group was close to that observed in 2005 among participants aged 25-34 years $^{(6)}$. The already high prevalence of overweight and obesity among some subgroups of young adults shows that further increases in the burden of overweight and obesity may be expected in the coming years.

Mean waist circumference increased between 2005 and 2014/2015. When compared with other sub-Saharan African countries, the mean values observed in Mozambique are lower than those reported for Benin, Kenya and Swaziland, both in women and men ${ }^{(27)}$. Although waist circumference is recognized as an important indicator complementing BMI to identify individuals at increased risk of obesityrelated morbidity ${ }^{(9)}$, the most common cut-offs used throughout the world to define abdominal obesity were based on a population of predominantly European origin $^{(28)}$. In fact, it was previously shown that black women from South Africa have less abdominal adipose tissue at the same waist circumference than European women $^{(29)}$. Therefore, in the absence of ethnic-specific cut-off points to define abdominal obesity in this setting, caution is needed when discussing prevalence data of abdominal obesity. Still, the prevalence, as defined using the cut-offs adopted by the $\mathrm{WHO}^{(9)}$, more than doubled among women in just a decade and so efforts need to be made to understand its possible impact on health outcomes, particularly on cardiometabolic diseases.

Similar to the findings from the first WHO STEPS survey conducted in Mozambique ${ }^{(6)}$, our results showed a higher prevalence of overweight and obesity in urban settings, both among women and men. A higher prevalence of overweight and obesity in urban areas was also reported in other sub-Saharan African countries such as Malawi ${ }^{(30)}$ or Uganda ${ }^{(31)}$, while equal or higher prevalence has been found in rural areas of European countries ${ }^{(32)}$ and the $\mathrm{USA}^{(33)}$. At the same time, the gap between the prevalence of underweight and overweight/obesity has increased sharply as expected in countries under epidemiological and nutrition transitions.

Although in the 2005 survey the prevalence of overweight and obesity tended to increase with education, both in rural and urban areas ${ }^{(6)}$, in 2014/2015 the association between obesity and education was weaker. This may reflect a more advanced stage of the transition than that found in the earlier survey, as in the more advanced stages of the obesity transition there is an inverse relationship between socio-economic status and obesity prevalence ${ }^{(7)}$.

The present study was based on a large representative sample of the adult population, and it allowed a direct comparison with a previous survey conducted in Mozambique, using essentially the same standardized procedures and following the WHO STEPS methodology. However, some limitations need to be discussed. Participants for whom data were unavailable on anthropometric measures were excluded from the current analysis. Nevertheless, given the low proportion of participants with missing data and the fact that they were not significantly different from those included in the analysis regarding gender, age or education, the impact of their exclusion on the validity of our results is expected to be low. Also, despite the large overall sample size, the precision of the estimates obtained for some subgroups considered in the stratified analyses was lower than desirable, especially among rural men. The lack of data in the 2005 and 2014/2015 STEPS surveys for younger children and adolescents precludes the study of the prevalence of overweight and obesity among these age groups, which would provide a better understanding of the burden expected for the next years in this population.

\section{Conclusion}

To conclude, in Mozambique there was a steep increase in the prevalence of overweight and obesity among adults in the past 10 years. The prevalence of obesity and overweight is higher in urban areas and in women, already affecting one in every four urban women aged $18-24$ years. These results suggest that culturally adapted interventions are needed to tackle the increasing morbidity and mortality burden that may be expected in the next years in Mozambique, especially among women and urban dwellers with higher socio-economic status.

\section{Acknowledgements}

Financial support: This study was supported by the Mozambican Ministry of Health and by the WHO. The study design and data collection were performed according to WHO guidelines. In addition, this study was funded by FEDER through the Operational Programme Competitiveness and Internationalization and national funding from 
the Foundation for Science and Technology - FCT (Portuguese Ministry of Science, Technology and Higher Education) under the 'Unidade de Investigação em Epidemiologia - Instituto de Saúde Pública da Universidade do Porto (EPIUnit)' (POCI-01-0145-FEDER006862; ref. UID/DTP/04750/2019). The funders had no role in the design, analysis or writing of this article. Conflict of interest: None. Authorship: A.D., C.S.-M. and N.L. conceived and designed the study. F.F. and N.L. analysed data. All authors were responsible for data interpretation. F.F. wrote the first version of the manuscript. A.D., N.J., A.P., C.S.-M., P.P. and N.L. critically revised the manuscript for relevant intellectual content. All authors approved the final version for submission. Ethics of human subject participation: This study was conducted according to the guidelines laid down in the Declaration of Helsinki and all procedures involving human subjects were approved by the National Bioethics Committee for Health. Written informed consent was obtained from all subjects.

\section{References}

1. Popkin BM, Adair LS \& Ng SW (2012) Global nutrition transition and the pandemic of obesity in developing countries. Nutr Rev 70, 3-21.

2. Development Initiatives (2018) Global Nutrition Report: Shining a Light to Spur Action on Nutrition. Bristol: Development Initiatives.

3. Haslam DW \& James WP (2005) Obesity. Lancet 366, 1197-1209.

4. Afshin A, Forouzanfar MH, Reitsma MB et al. (2017) Health effects of overweight and obesity in 195 countries over 25 years. $N$ Engl J Med 377, 13-27.

5. World Health Organization (2017) WHO STEPS Surveillance Manual: The WHO STEPwise approach to noncommunicable disease risk factor surveillance. http://www.who.int/ chp/steps/manual/en/ (accessed January 2018).

6. Gomes A, Damasceno A, Azevedo A et al. (2010) Body mass index and waist circumference in Mozambique: urban/rural gap during epidemiological transition. Obes Rev 11, 627-634.

7. Jaacks LM, Vandevijvere S, Pan A et al. (2019) The obesity transition: stages of the global epidemic. Lancet Diabetes Endocrinol 7, 231-240.

8. Jessen N, Damasceno A, Silva-Matos C et al. (2018) Hypertension in Mozambique: trends between 2005 and 2015. J Hypertens 36, 779-784.

9. World Health Organization (2011) Waist Circumference and Waist-Hip Ratio. Report of a WHO Expert Consultation, Geneva, 8-11 December 2008. Geneva: WHO.

10. Instituto Nacional de Estatística (n.d.) Recenseamento Geral da População e Habitação. http://www.ine.gov.mz/ operacoes-estatisticas/censos/censo-2007 (accessed January 2018).

11. Sunghee L, William WD, Hoang AN et al. (2007) Examining trends and averages using combined cross-sectional survey data from multiple years. CHIS Methodology Paper. https://healthpolicy.ucla.edu/chis/faq/Documents/paper_ trends_averages.pdf (accessed January 2018).

12. $\mathrm{Ng} \mathrm{M}$, Fleming T, Robinson M et al. (2014) Global, regional, and national prevalence of overweight and obesity in children and adults during 1980-2013: a systematic analysis for the Global Burden of Disease Study 2013. Lancet 384, 766-781.

13. Kelly T, Yang W, Chen CS et al. (2008) Global burden of obesity in 2005 and projections to 2030. Int J Obes (Lond) 32, 1431-1437.

14. Amugsi DA, Dimbuene ZT, Mberu B et al. (2017) Prevalence and time trends in overweight and obesity among urban women: an analysis of demographic and health surveys data from 24 African countries, 1991-2014. BMJ Open 7, e017344.

15. Abubakari AR, Lauder W, Agyemang C et al. (2008) Prevalence and time trends in obesity among adult West African populations: a meta-analysis. Obes Rev 9, 297-311.

16. NCD Risk Factor Collaboration (NCD-RisC) - Africa Working Group (2017) Trends in obesity and diabetes across Africa from 1980 to 2014: an analysis of pooled population-based studies. Int J Epidemiol 46, 1421-1432.

17. The World Bank (2018) World Development Indicators. https://data.worldbank.org/ (accessed January 2018).

18. Instituto Nacional de Estatística (n.d.) Projecções da População: 2007-2040. http://www.ine.gov.mz/estatisticas/ estatisticas-demograficas-e-indicadores-sociais/projeccoes-dapopulacao (accessed January 2018).

19. Padrao P, Damasceno A, Silva-Matos C et al. (2012) Physical activity patterns in Mozambique: urban/rural differences during epidemiological transition. Prev Med 55, 444-449.

20. World Health Organization (2005) Chronic diseases and health promotion. Global physical activity surveillance. www.who.int/chp/steps/GPAQ/en/index.html (accessed January 2018).

21. Instituto Nacional de Estatística (2015) Relatório Final do Inquérito ao Orçamento Familiar - IOF 2014/15. Maputo: Instituto Nacional de Estatística.

22. dos Santos FK, Maia JA, Gomes TN et al. (2014) Secular trends in habitual physical activities of Mozambican children and adolescents from Maputo City. Int J Environ Res Public Health 11, 10940-10950.

23. Ministério de Economia e Finanças (2016) Pobreza e bemestar em Moçambique: Quarta avaliação nacional. Inquérito ao Orçamento Familiar - IOF 2014/15. http:// igmozambique.wider.unu.edu/ (accessed January 2018).

24. Food and Agriculture Organization of the United Nations (2011) Nutrition country profile: Republic of Mozambique. http://www.fao.org/3/ap844e/ap844e.pdf (accessed January 2018).

25. Padrao P, Laszczynska O, Silva-Matos C et al. (2012) Low fruit and vegetable consumption in Mozambique: results from a WHO STEPwise approach to chronic disease risk factor surveillance. Br J Nutr 107, 428-435.

26. Nelson MC, Story M, Larson NI et al. (2008) Emerging adulthood and college-aged youth: an overlooked age for weight-related behavior change. Obesity (Silver Spring) 16, 2205-2211.

27. World Health Organization (2016) STEPS Country Reports. http://www.who.int/ncds/surveillance/steps/reports/en/ (accessed January 2018).

28. Han TS, van Leer EM, Seidell JC et al. (1995) Waist circumference action levels in the identification of cardiovascular risk factors: prevalence study in a random sample. $B M J$ 311, 1401-1405.

29. Rush EC, Goedecke JH, Jennings C et al. (2007) BMI, fat and muscle differences in urban women of five ethnicities from two countries. Int J Obes (Lond) 31, 1232-1239.

30. Price AJ, Crampin AC, Amberbir A et al. (2018) Prevalence of obesity, hypertension, and diabetes, and cascade of care in sub-Saharan Africa: a cross-sectional, population-based study in rural and urban Malawi. Lancet Diabetes Endocrinol 6, 208-222. 
31. Kirunda BE, Fadnes LT, Wamani H et al. (2015) Populationbased survey of overweight and obesity and the associated factors in peri-urban and rural Eastern Uganda. BMC Public Health 15, 1168.

32. Peytremann-Bridevaux I, Faeh D \& Santos-Eggimann B (2007) Prevalence of overweight and obesity in rural and urban settings of 10 European countries. Prev Med 44, 442-446.

33. Befort CA, Nazir N \& Perri MG (2012) Prevalence of obesity among adults from rural and urban areas of the United States: findings from NHANES (2005-2008). J Rural Health 28, 392-397. 